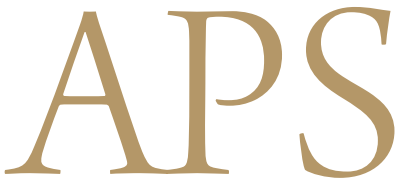

Archives of Plastic Surgery

\title{
Polyurethane-Coated Breast Implants Revisited: A 30-Year Follow-Up
}

\author{
Nikki Castel, Taylor Soon-Sutton, Peter Deptula, Anna Flaherty, Fereydoun Don Parsa \\ Department of Surgery, Division of Plastic Surgery, University of Hawai'i, John A. Burns School of Medicine, Honolulu, HI, USA
}

Background Polyurethane coating of breast implants has been shown to reduce capsular contracture in short-term follow-up studies. This 30-year study is the longest examination of the use of polyurethane-coated implants and their correlation with capsular contracture.

Methods This study evaluates the senior surgeon's (F.D.P.) experience with the use of polyurethanecoated implants in aesthetic breast augmentation in 382 patients over 30 years. Follow-up evaluations were conducted for six months after surgery. After the six-month follow-up period, 76 patients returned for reoperation. The gross findings, histology, and associated capsular contracture were noted at the time of explantation.

Results No patient during the six-month follow-up period demonstrated capsular contracture. For those who underwent reoperation for capsular contracture, Baker II/III contractures were noted nine to 10 years after surgery and Baker IV contractures were noted 12 to 21 years after surgery. None of the explanted implants had macroscopic evidence of polyurethane, which was only found during the first five years after surgery. The microscopic presence of polyurethane was noted in all capsules up to 30 years after the original operation.

Conclusions An inverse correlation was found between the amount of polyurethane coating on the implant and the occurrence of capsular contracture. Increasingly severe capsular contracture was associated with a decreased amount of polyurethane coating on the surface of the implants. No contracture occurred in patients whose implants showed incomplete biodegradation of polyurethane, as indicated by the visible presence of polyurethane coating. We recommend research to find a non-toxic, non-biodegradable synthetic material as an alternative to polyurethane.

Keywords Polyurethanes / Implant capsular contracture / Breast implants

Received: 13 Nov 2014 • Revised: 6 Dec 2014 • Accepted: 9 Dec 2014

pISSN: 2234-6163・elSSN: 2234-6171 • http://dx.doi.org/10.5999/aps.2015.42.2.186・Arch Plast Surg 2015;42:186-193

\author{
Correspondence: \\ Fereydoun Don Parsa \\ Department of Surgery, Division of \\ Plastic Surgery, University of Hawai'i, \\ John A. Burns School of Medicine, \\ 1329 Lusitania Street, Suite 807, \\ Honolulu, HI 96813, USA \\ Tel: +1-808-526-0303 \\ Fax: +1-808-536-8836 \\ E-mail:fdparsa@gmail.com
}

No potential conflict of interest relevant to this article was reported.

\section{INTRODUCTION}

Dow Corning Corporation manufactured the first silicone gel breast implant in 1962. In the 1970s, a second-generation silicone gel implant was introduced. The new implants were made of silicone, silicone gel, and urethane, and were designed to achieve a natural, safe, and pleasing result that the previous pros- theses had failed to achieve [1]. However, in 1988 the Food and Drug Administration (FDA) classified silicone and saline implants as Class III devices, corresponding to the highest level of risk. The FDA required manufacturers of silicone gel-filled breast implants to submit pre-market approval applications with data providing a reasonable assurance of the safety and effectiveness of the implants [2]. 
In 1991, Surgitek, a manufacturer of polyurethane-coated breast implants, voluntarily withdrew these implants from the market in response to public safety concerns regarding 2,4-toluenediamine (2,4-TDA), a breakdown product of polyurethane [3]. Studies of 2,4-TDA in animal models raised questions concerning the carcinogenicity of polyurethane-coated breast implants. The National Cancer Institute exposed rats and mice to 2,4-TDA through feeding. Resulting malignancies in these models were tied to the 2,4-TDA exposure [4]. It was later discovered that these experiments administered supra-physiological levels of 2,4-TDA to the rat and mice models [5]. Meanwhile, new evidence of polyurethane biodegradation emerged, which prompted further concern and investigation [6].

When the FDA reported laboratory findings of 2,4-TDA release from degraded polyurethane foam, Bristol-Myers Squibb discontinued their Meme and Replicon polyurethane-coated implants [5]. In June 1991, the FDA requested that Bristol-Myers Squibb investigate exposure to 2,4-TDA following polyurethane degradation in coated implants [2,5]. During the same time period, an FDA advisory panel determined that the risk of cancer associated with polyurethane was small and outweighed by the risks associated with removal of the breast implants [2]. Bristol-Myers Squibb enrolled subjects with polyurethane-coated implants, 16 to 102 months post-implantation, with an equal number of control subjects [5]. Hester et al. [6] examined the concentrations of polyurethane breakdown products in serum and urine samples in both groups. No subjects were found to have free 2,4-TDA, 2,6-TDA, or 4-acetyl TDA in serum samples. Urine analysis of the implant group revealed quantifiable amounts of free 2,4-TDA in 30 subjects and detectable amounts in 18. Urine analysis of the control group revealed no subjects with quantifiable amounts of free 2,4-TDA and seven subjects with detectable amounts [6]. Taking into account that TDA has a half-life of 21 months, the authors established an upper limit for the lifetime theoretical risk of cancer of 1.1 in one million for a single pair of polyurethane coated implants [6]. This estimate assumes that 2,4-TDA is carcinogenic in humans, which has never been proven [6].

Using the Bristol-Myers Squibb study as a reference, the FDA released a statement in 1995 reaffirming its conclusion on the safety of polyurethane-coated implants. The FDA stated that based on this study, the risk of developing cancer from polyurethane-coated implants is negligible [5].

Several studies have been conducted regarding the safety of polyurethane-coated breast implants without finding evidence of a causal relationship to malignancy or toxicity. These studies also report that the use of polyurethane-coated breast implants results in a superior aesthetic outcome with greater patient satis- faction [7-22]. Polyurethane-coated implants have also been associated with a lower risk of long-term complications such as rupture, ptosis, bleeding, infection, and contracture, the last of which is the focus of this paper.

Although the exact mechanism that leads to capsular contracture is unknown, research suggests that several factors influence the incidence of contracture, including biofilms, infections, hematoma, irradiation, smoking, implant surface, subglandular placement, the postoperative use of a surgical bra, and a periareolar incision site. Based on the Kaplan-Meier multivariate analysis, the two strongest contributing factors for capsular contracture are smooth surface implants and subglandular placement. Additionally, the device size is inversely proportional to the risk of developing contracture: increased size is associated with a decreased risk of capsular contracture. Inframammary incisions have resulted in reduced contracture rates, but are not advisable for Grade I and II tubular or ptotic breast shapes less than $200 \mathrm{~g}$ in size, or when the inframammary crease is nonexistent or high [23].

Histologic studies of contractures around all types of implants reveal that contracture is associated with a foreign body reaction in which macrophages are predominant. In smooth layer implants, a dense monolayer of inflammatory cells surrounds the foreign body surface. Over time, the macrophages secrete substances that attract fibroblasts and encourage them to proliferate. The fibroblasts produce collagen, which produces circumferential contracture when placed under tension by the fibrous tissue. A polyurethane coating, however, rids implants of their smooth layer. Microscopic analyses have shown that polyurethane produces an irregular, interconnected coating that resembles a spongy meshwork, allowing the foreign body reaction to fill the spaces in the meshwork. Instead of creating a single longitudinal collagen capsule, as in smooth implants, the collagen must be deposited along the irregular spongy shape of the coating. The tension from the fibrous tissue is thus spread around the implant as a whole instead of extending in one linear direction, reducing the incidence of contracture [24].

Another factor leading to a decreased incidence of contracture in polyurethane-coated implants is the biodegradation of polyurethane, which causes it to fragment. The fragments are phagocytized by macrophages and produce the same foreign body reaction described above, forming microcapsules. Each microcapsule is individually enveloped in fibrotic tissue, preventing the organized alignment of myofibroblasts, which in turn prevents the alignment of force vectors needed for the development of capsular contracture. Although this property of polyurethane coating contributes to the decreased incidence of contracture, it also increases the rate of degradation. In most cases, the poly- 
urethane foam coating begins to disappear about two years after surgery [24].

Our goal in this study was to evaluate the long-term complications of polyurethane-coated breast implants, focusing primarily on capsular contracture and its correlation with the presence of polyurethane coating on the surface of the prosthesis at the time of explantation.

\section{METHODS}

\section{Surgical technique}

All procedures were primary breast augmentations performed by the same senior surgeon (F.D.P.) or under his direct supervision. Only polyurethane-coated implants from Bristol-Myers Squibb were used in this study. Half of the implants were round (Meme) and the others were anatomical implants (Replicon). The polyurethane coating was identical in both round and anatomical implants. All devices were placed through inframammary incisions.

\section{Infection prophylaxis}

A strict no-touch protocol was utilized for all patients. All pockets were irrigated with a 5\% solution of betadine (povidone-iodine) before implant insertion. Closed-tube drainage was employed in all patients, and the tubes were removed within two to five days. Intravenous cephalosporin was administered as a single dose at the start of the operation. No additional antibiotics were provided thereafter.

\section{Patient population}

We reviewed the records from 1982 to 2013 of patients who had undergone breast augmentation with polyurethane-coated sili-

\section{Fig. 1. Anterior view eight years post-surgery}

A patient is shown demonstrating no contracture eight years after submuscular augmentation with $350 \mathrm{~mL}$ round Méme implants, placed through inframammary incisions.

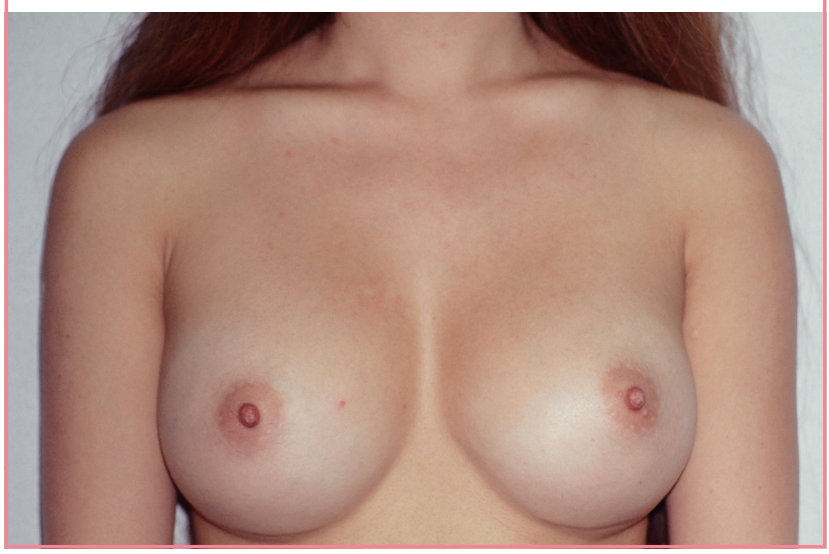

cone gel implants. From 1982 until their discontinuation in 1991, 764 polyurethane implants were inserted in 382 patients. All patients were asked to return for follow-up visits at one week, four weeks, six weeks, and six months after surgery.

\section{Evaluation}

After six months of follow-up, 76 patients returned to the senior surgeon's clinic at various intervals for reoperation over a 30year period. Photographs of the implants were taken at the time of explantation and capsules were submitted for microscopic examination. Indications for reoperation, intraoperative findings, microscopic findings, and factors correlating with capsular contracture at the time of reoperation were documented. The degree of capsular contracture was recorded based on the Baker scale: Baker I indicates no contracture, Baker II/III refers to mild to moderate contracture with no breast deformity, and Baker IV indicates severe contracture with a visible breast deformity. In patients who had contracture on only one side, or if one breast showed less contracture, the side with the higher degree of contracture was reported.

\section{RESULTS}

From 1981 to 1991,764 polyurethane-coated silicone gel implants were inserted in 382 patients. Of this group, all 382 patients were seen at a one-week follow-up, 378 were seen at four weeks; 322 were seen at six weeks, and 288 were seen at six months after surgery. No patient during this follow-up period experienced capsular contracture. A normal postoperative patient eight years after surgery experienced no capsular contracture, as shown in Figs. 1 and 2.

Seventy-six patients (19.9\%) underwent reoperation based on various indications, as shown in Table 1 . The indications for re-

Fig. 2. Lateral view eight years post-surgery

A lateral view is shown of the same patient as in Fig. 1.

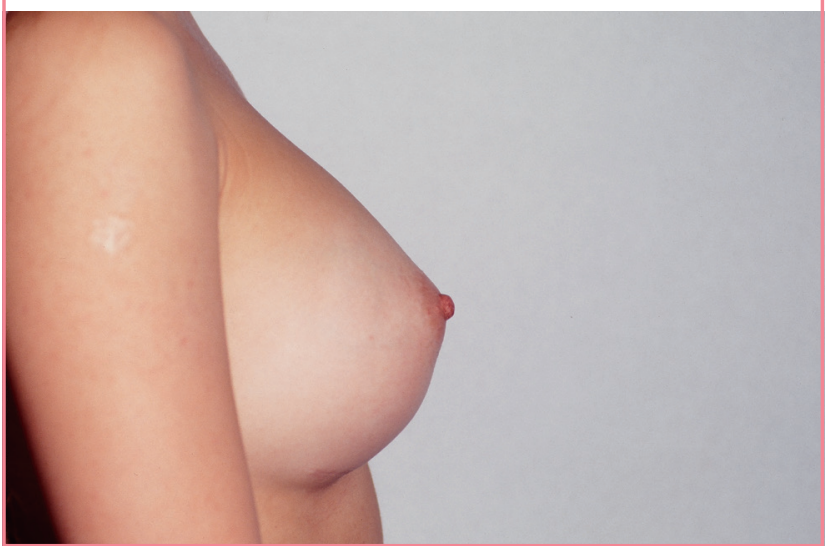




\section{Table 1. Indications for reoperation}

\begin{tabular}{|c|c|c|}
\hline Reason for reoperation & $\begin{array}{l}\text { No. of patients } \\
(n=82)\end{array}$ & $\begin{array}{l}\text { Percent with a given } \\
\text { complication (\%) }\end{array}$ \\
\hline $\begin{array}{l}\text { Desire to exchange implant } \\
\text { for saline prosthesis }\end{array}$ & 51 & 13.4 \\
\hline Capsular contracture: total & 9 & 2.4 \\
\hline $\begin{array}{l}\text { Capsular contracture: } \\
\text { Baker II/III }\end{array}$ & 2 & 0.5 \\
\hline $\begin{array}{l}\text { Capsular contracture: } \\
\text { Baker IV }\end{array}$ & 7 & 1.8 \\
\hline Ruptured implant: suspected & 12 & 3.1 \\
\hline $\begin{array}{l}\text { Ruptured implant: found at } \\
\text { exploration }\end{array}$ & 9 & 2.4 \\
\hline $\begin{array}{l}\text { Immediate hematoma } \\
\text { (within } 24 \mathrm{hr} \text { ) }\end{array}$ & 5 & 1.3 \\
\hline Delayed bleed & 2 & 0.5 \\
\hline Infectiona) & 1 & 0.3 \\
\hline $\begin{array}{l}\text { Ptosis and displaced } \\
\text { inframammary fold }\end{array}$ & 2 & 0.5 \\
\hline $\begin{array}{l}\text { Removed without } \\
\text { replacement }\end{array}$ & 16 & 4.2 \\
\hline \multicolumn{3}{|c|}{$\begin{array}{l}\text { The number of patients who developed complications requiring reoperation and } \\
\text { the resulting } 30 \text {-year complication rates are shown, following initial breast } \\
\text { augmentation surgery in } 382 \text { patients. } \\
\text { a) No evidence of actual infection after explantation. Erythema was secondary to } \\
\text { inflammatory response to polyurethane. }\end{array}$} \\
\hline
\end{tabular}

operation were as follows: the desire to change polyurethanecoated implants to saline prostheses of the same or a different size (51 patients), capsular contracture ( 9 patients) (Baker II/ III, 2; Baker IV, 7), a suspected ruptured implant (12 patients, of whom 9 were confirmed as having ruptured implants), immediate hematoma ( 5 patients), delayed bleeding ( 2 patients), capsular ptosis ( 2 patients), infection ( 1 patient), and the desire to remove implants without a replacement (16 patients). Patients who requested replacements after the removal of the polyurethane implants were given various replacements over the period encompassed by this study. Before 1992, gel implants, including polyurethane-coated implants, were used. In 1992, all gel implants were taken off the market in the United States, and therefore textured saline implants were used between 1992 and 2006. The FDA reapproved the use of gel implants in 2006, after which textured gel implants were exclusively used.

Among the 288 patients (75.4\%) who returned for their sixmonth follow-up visit, soft breasts (Baker I) were noted in all patients. Of the 76 patients who eventually underwent reoperation, as described in Table 1, all patients demonstrated soft breasts with no contracture (Baker I) at their six-month follow-up visit. After this period, 67 patients continued to show no contracture (Baker I) and nine patients experienced capsular contracture of different degrees. Baker II/III contracture was noted in two of the nine patients at nine and 10 years after surgery. One of the patients with grade III contracture is shown 29 years after surgery in Figs.
Fig. 3. Anterior view 29 years post-surgery with grade III contracture

A patient is shown demonstrating grade III contracture 29 years after subglandular augmentation with $400 \mathrm{~mL}$ Replicon implants, placed through inframammary incisions.

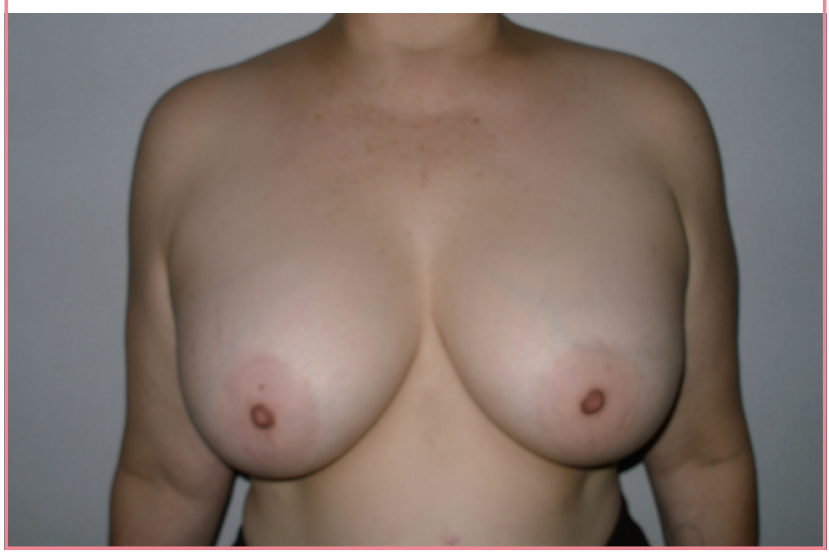

Fig. 4. Lateral view 29 years post-surgery

A lateral view is shown of the same patient as in Fig. 3.

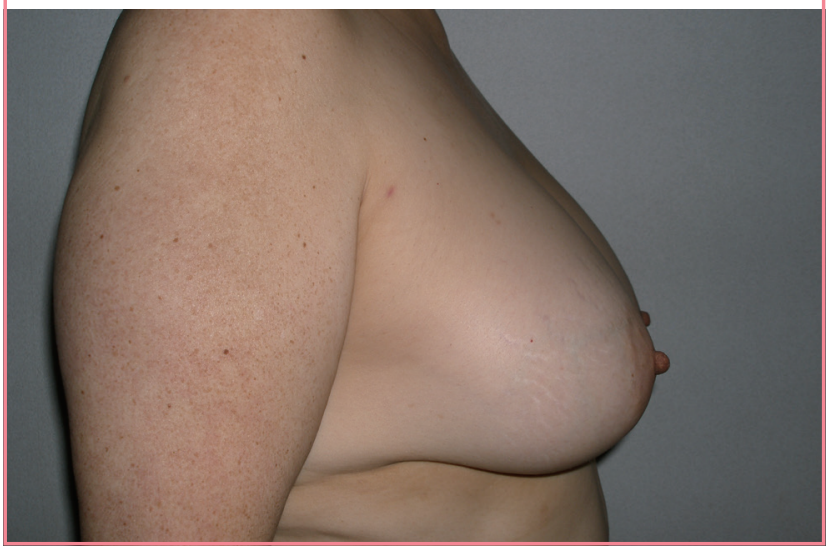

3 and 4 . The remaining seven patients were determined to have Baker IV contracture at office visits that occurred 12, 13, 14, 15, 17,18 , and 21 years after surgery, with a mean follow-up time of 16 years.

Macroscopic evidence of polyurethane was noted on the surface of implants upon explantation up to five years after the original operation (Fig. 5). Beginning at six years after the operation, the polyurethane gradually disintegrated and eventually disappeared, as shown in Fig. 6. In contrast, polyurethane was present microscopically within all capsules during the entire observation period of 30 years.

Histologic studies of removed capsules revealed vacuoles and indications of a foreign body reaction (Fig. 7). Although no specific laboratory test is available to determine whether polyurethane was the source of these changes, we assume that these findings were the result of polyurethane material embedded in 
Fig. 5. Polyurethane implants four to five years postsurgery

Explantation up to five years after breast augmentation surgery demonstrates that macroscopic remnants of polyurethane are present on the surface of all implants.

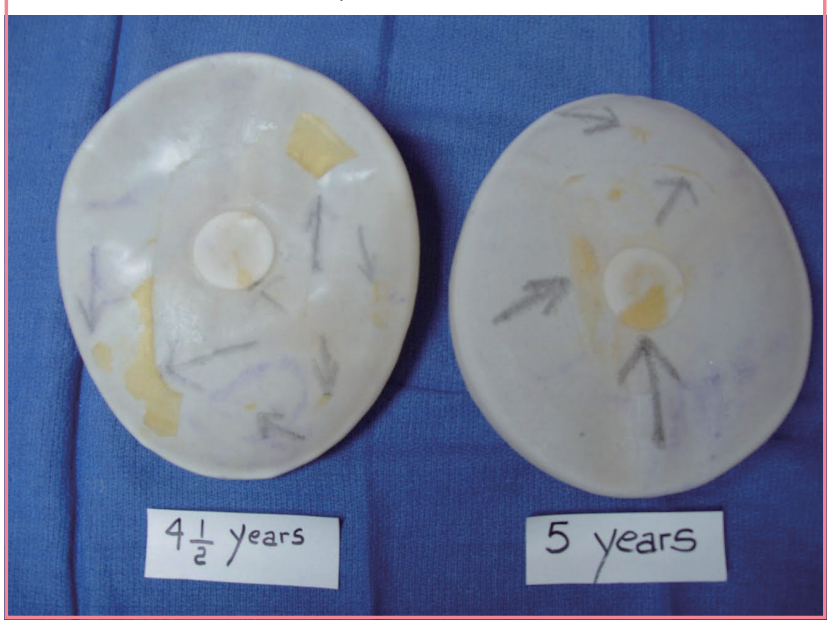

Fig. 6. Polyurethane implants six to 10 years post-surgery

Beginning at six years after the operation, no macroscopic presence of polyurethane on the surface of implants can be observed. This is also demonstrated at eight and 10 years after the operation.

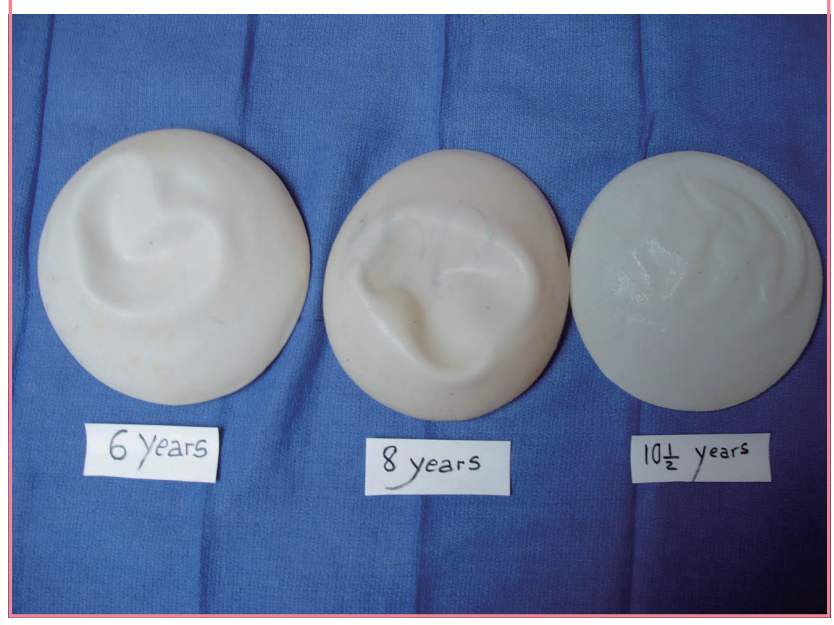

capsular tissue.

Immediate postoperative bleeding within six to 12 hours after surgery occurred in five patients (1.3\%). These cases required exploration and evacuation of the hematoma within 24 hours. All cases were unilateral. None had predisposing causes such as hypertension or coagulopathy. None of these patients developed capsular contracture during their follow-up visits with the senior surgeon and no colleagues who may have later seen these patients reported any incidence of capsular contracture.

Late hematomas requiring exploration occurred in two patients $(0.5 \%)$ : one at nine years and one at 23 years. The causes of bleeding were unknown. The sole case of late hematoma oc-

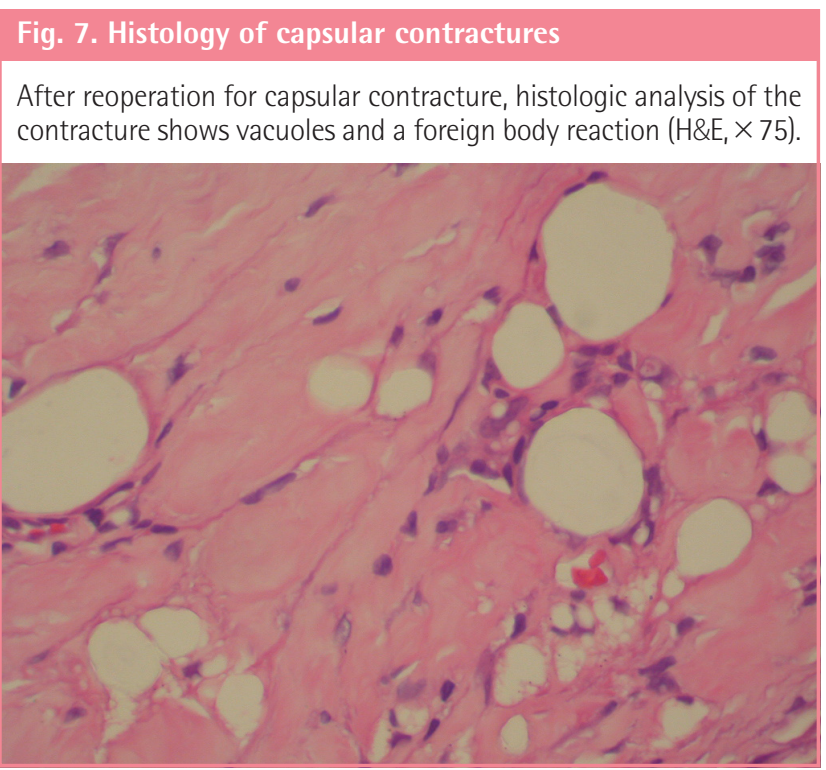

Fig. 8. Capsular ptosis

Left breast capsular ptosis after submuscular breast augmentation. The implant was completely detached from its coating.

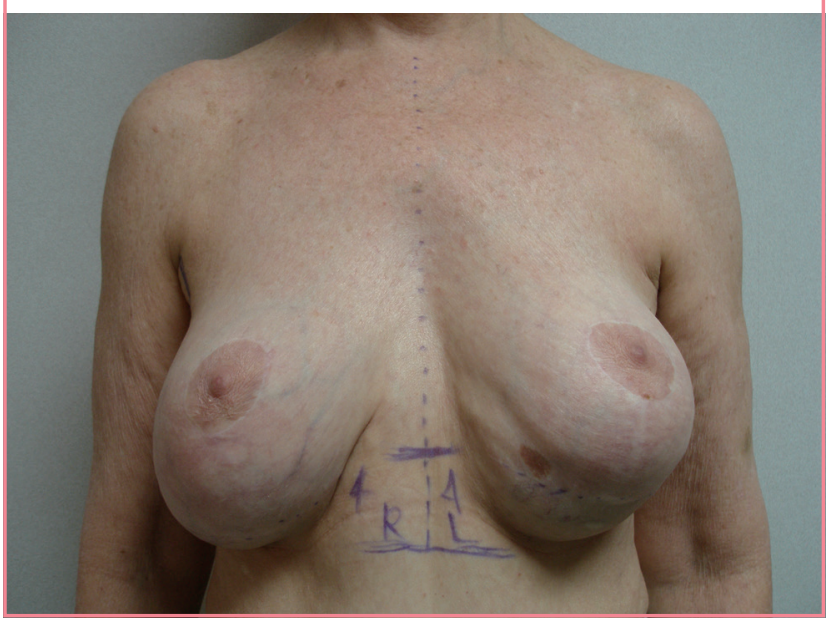

curring nine years after augmentation mammoplasty, with photographs of the complication, was previously reported in the Journal of Aesthetic Plastic Surgery in 2004. This case of late hematoma was thought to be due to chronic inflammation in response to the polyurethane [25].

One patient was treated for infection of both breasts in 1983, during the early period of our experience with the use of polyurethane implants. Exploration was carried out because of erythema noted on postoperative day four. Despite antibiotic treatment, the erythema progressed to involve the entirety of both breasts by postoperative day six. Although no fever or other systemic symptoms were present, the patient was explored for possible bilateral implant infection on postoperative day nine. No gross evidence of infection was noted. Gram staining and cul- 


\section{Fig. 9. Capsulorrhaphy technique}

This image demonstrates the capsulorrhaphy technique used on the patient in Fig. 8 to correct her capsular ptosis.

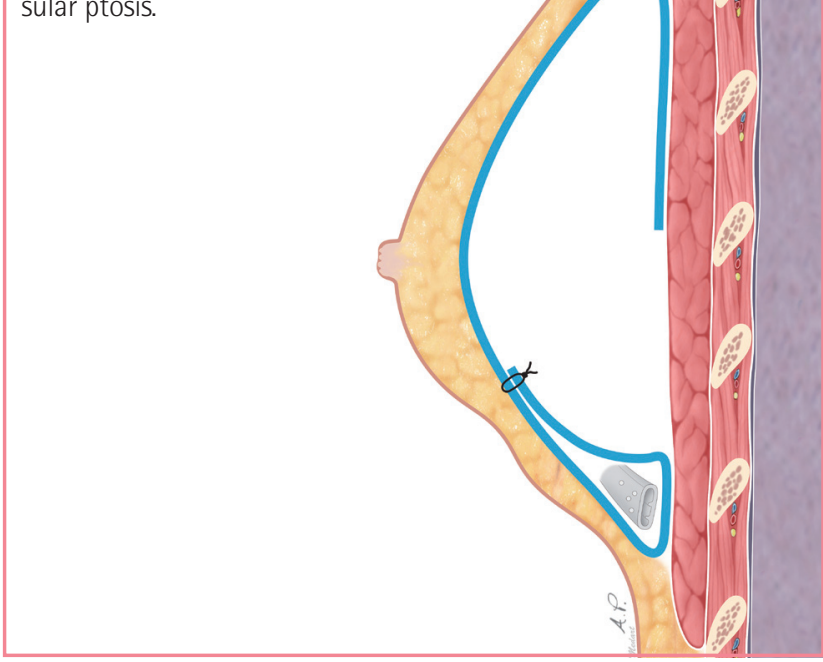

tures were negative. The patient did not develop any infection, capsular contracture, or other problems during her six-month follow-up period.

With more experience in the use of these implants, we learned that erythema commonly occurs after polyurethane implantation. This may be secondary to an inflammatory response to polyurethane. The erythema subsides spontaneously with no treatment. Erythema of this type was recorded in 93 patients $(24.3 \%)$ and was bilateral in all cases. The degree and extent of erythema varied in each breast. No fever, pain, tenderness, or swelling accompanied the erythema. These findings were typically noted on the third to fifth day after implantation and subsided gradually within two to three weeks.

Capsular ptosis with inferior displacement of the implants occurred in two patients. In both cases, the ptosis was unilateral. In one patient, the implant was completely detached from its surrounding capsule on the ptotic side. This patient is shown in Fig. 8. The capsulorrhaphy technique, demonstrated in Fig. 9, was utilized to correct this condition [8]. In the other patient, however, the polyurethane remained attached to the capsule. The mechanism of this case of ptosis is obscure since it was not present during the initial follow-up period $[7,8]$.

\section{DISCUSSION}

Our study shows a $2.4 \%$ incidence of contracture (Baker II-IV) during this extended follow-up period. Other studies show a wide range of capsular contracture incidence, ranging from $0.98 \%$ over 10 years to $30 \%$ over 25 years [9-15]. These rates of contracture

\section{Fig. 10. Capsular contracture after surgery}

Before the disappearance of macroscopic polyurethane, no cases of capsular contracture were noted. Beginning at three to four years after the disappearance of macroscopic polyurethane, Baker II, III, and IV contractures developed, with increasing incidence thereafter.

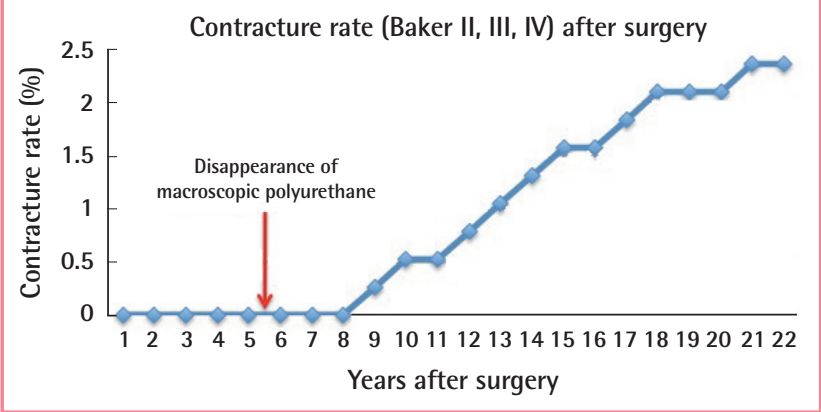

in polyurethane-coated implants are significantly lower than those reported for smooth implants. Coleman et al. [15] reported an $8 \%$ contracture rate for non-polyurethane textured implants compared to $58 \%$ for smooth implants after 12 months of follow-up.

We postulate that the significantly lower rate of capsular contracture observed with polyurethane-coated breast implants may be attributed to a high degree of adhesiveness to the adjacent tissues, due to both the particular material used in the coating and the microscopic structure of the surface.

The polyurethane coating was examined macroscopically after explantation up to five years after surgery. The earliest incidence of capsular contracture (Baker II/III) was noted nine years after surgery. This observation correlates with our overall observation that polyurethane gradually degrades from five to nine years after implantation. Beyond nine years after implantation, no coating was present in any patient, which is when we noted an increasing incidence and severity of contracture. This observation demonstrates a distinct correlation between the gradual disappearance of polyurethane coating and both the occurrence and severity of capsular contracture (Fig. 10).

The suspected rupture rate in this study before exploration was $3.1 \%$ while the observed rupture rate was $2.4 \%$. Other studies show rupture rates below $6 \%$, similar to ours [11-17].

Unlike contracture and rupture rates, the infection rates reported in the literature do not vary widely. The reported rates are relatively low, ranging from $1.1 \%$ to $3.4 \%[11,12,14,16]$. We observed a $0.3 \%$ rate of suspected infections that required exploration, but no patient developed a clinical infection that required explantation. The protocol for infection prophylaxis is discussed in the materials and methods section.

Postoperative bleeding occurred in $1.3 \%$ of patients within six to 12 hours of surgery and $0.5 \%$ developed a late hematoma, at 
nine and 23 years after surgery, respectively. Thus, the total bleeding complication rate was $1.8 \%$. These rates are similar to those reported by other authors [12-17].

The main limitation of this study relates to return visits by patients. The complication rates reported in this study are contingent upon patients returning for follow-up visits. Some patients may have left the state or undergone reoperation by other surgeons without our knowledge. Therefore, the data presented in this study may not reflect the exact incidence of re-exploration.

In summation, this 30-year study is the longest investigation of the clinical and histologic outcomes following breast augmentation with polyurethane-coated implants. We found that polyurethane-coated breast implants are associated with significantly less risk of capsular contracture compared to other types of implants, including both smooth and textured surface implants. The presence of macroscopic polyurethane coating on the surface of implants correlated directly with a $0 \%$ incidence of capsular contracture. As the coating gradually disintegrated, typically starting around five years after implantation, capsular contracture began to occur. Soft breasts (Baker I) were always correlated with presence of the polyurethane coating. In contrast, when no coating was macroscopically observable at the time of exploration, various degrees of capsular contracture were noted. Although macroscopic evidence of polyurethane was absent after five years, polyurethane material was microscopically documented within the capsules of all patients up to 30 years after surgery. We postulate that the long-term presence of microscopic polyurethane material may contribute to the lower incidence of contracture in polyurethane-coated implants compared to non-coated implants.

Polyurethane implants have not been reintroduced in the US since their discontinuation in 1992. Studies have shown potential toxicity with polyurethane and our studies have shown that it tends to disintegrate. However, due to its superior aesthetic outcome and significantly lower rates of capsular contracture, we encourage further studies investigating alternative non-biodegradable and non-toxic materials similar in structure to polyurethane. A non-biodegradable material would coat implants for an extended period of time, which would further decrease the incidence of capsular contracture and perhaps eliminate it altogether.

\section{REFERENCES}

1. Ashley FL. A new type of breast prosthesis. Preliminary report. Plast Reconstr Surg 1970;45:421-4.

2. U.S. Food and Drug Administration. FDA breast implant consumer handbook 2004: timeline of breast implant activi- ties. Silver Spring, MD: U.S. Food and Drug Administration; 2013 [cited 2015 Jan 12]. Available from: http://www. fda.gov/MedicalDevices/ProductsandMedicalProcedures/ ImplantsandProsthetics/BreastImplants/ucm064242.htm.

3. Van Zele D, Heymans O. Breast implants. A review. Acta Chir Belg 2004;104:158-65.

4. National Toxicology Program. Bioassay of 2,4-diaminotoluene for possible carcinogenicity. Natl Cancer Inst Carcinog Tech Rep Ser 1979;162:1-139.

5. Pharma and MedTech Business Intellignece. The gray sheet: bristol-myers squibb polyurethane-coated breast implant study [Internet]. Informa Business Information Inc.; 1993 [cited 2015 Jan 12]. Available from: https://www.pharmamedtechbi.com/publications/the-gray-sheet/19/040/bristolmyers-squibb-polyurethanecoated-breast-implant-study.

6. Hester TR Jr, Ford NF, Gale PJ, et al. Measurement of 2,4-toluenediamine in urine and serum samples from women with Meme or Replicon breast implants. Plast Reconstr Surg 1997; 100:1291-8.

7. Parsa FD. Displaced inframammary fold after Meme breast augmentation. Plast Reconstr Surg 1988;81:643-4.

8. Parsa FD. Breast capsulopexy for capsular ptosis after augmentation mammaplasty. Plast Reconstr Surg 1990;85:80912.

9. Collis N, Coleman D, Foo IT, et al. Ten-year review of a prospective randomized controlled trial of textured versus smooth subglandular silicone gel breast implants. Plast Reconstr Surg 2000;106:786-91.

10. Cohney BC, Mitchell S. An improved method of removing polyurethane foam-covered gel prostheses. Aesthetic Plast Surg 1997;21:191-2.

11. Henriksen TF, Fryzek JP, Holmich LR, et al. Reconstructive breast implantation after mastectomy for breast cancer: clinical outcomes in a nationwide prospective cohort study. Arch Surg 2005; 140:1152-9.

12. Kulmala I, McLaughlin JK, Pakkanen M, et al. Local complications after cosmetic breast implant surgery in Finland. Ann Plast Surg 2004;53:413-9.

13. Vazquez G. A ten-year experience using polyurethane-covered breast implants. Aesthetic Plast Surg 1999;23:189-96.

14. Handel N, Cordray T, Gutierrez J, et al. A long-term study of outcomes, complications, and patient satisfaction with breast implants. Plast Reconstr Surg 2006;117:757-67.

15. Coleman DJ, Foo IT, Sharpe DT. Textured or smooth implants for breast augmentation? A prospective controlled trial. Br J Plast Surg 1991;44:444-8.

16. Kjoller K, Holmich LR, Jacobsen PH, et al. Epidemiological investigation of local complications after cosmetic breast im- 
plant surgery in Denmark. Ann Plast Surg 2002;48:229-37.

17. Gabriel SE, Woods JE, O’Fallon WM, et al. Complications leading to surgery after breast implantation. $\mathrm{N}$ Engl J Med 1997;336:677-82.

18. Peters W, Keystone E, Smith D. Factors affecting the rupture of silicone-gel breast implants. Ann Plast Surg 1994;32:44951.

19. de Camara DL, Sheridan JM, Kammer BA. Rupture and aging of silicone gel breast implants. Plast Reconstr Surg 1993; 91:828-34.

20. Robinson OG Jr, Bradley EL, Wilson DS. Analysis of explanted silicone implants: a report of 300 patients. Ann Plast Surg 1995;34:1-6.

21. Marotta JS, Goldberg EP, Habal MB, et al. Silicone gel breast implant failure: evaluation of properties of shells and gels for explanted prostheses and meta-analysis of literature rupture data. Ann Plast Surg 2002;49:227-42.

22. Brown SL, Pennello G, Berg WA, et al. Silicone gel breast implant rupture, extracapsular silicone, and health status in a population of women. J Rheumatol 2001;28:996-1003.

23. Stevens WG, Nahabedian MY, Calobrace MB, et al. Risk factor analysis for capsular contracture: a 5-year Sientra study analysis using round, smooth, and textured implants for breast augmentation. Plast Reconstr Surg 2013;132:1115-23.

24. Brand KG. Foam-covered mammary implants. Clin Plast Surg 1988; 15:533-9.

25. Brickman M, Parsa NN, Parsa FD. Late hematoma after breast implantation. Aesthetic Plast Surg 2004;28:80-2. 